\title{
Can a Focus on Breakthrough Technologies Improve the Performance of International Environmental Agreements?
}

\author{
Michael Hoel · Aart de Zeeuw
}

Accepted: 13 May 2010 / Published online: 30 May 2010

(C) The Author(s) 2010. This article is published with open access at Springerlink.com

\begin{abstract}
In a recent paper, Barrett (Am Econ Rev 96(2):22-25, 2006) reaches the conclusion that in general the answer to the question in the title is no, except for a special case in which technology adoption involves increasing returns (network externalities). We show in this paper that a focus on the R\&D phase in the development of breakthrough technologies can also increase the possibilities for cooperation.
\end{abstract}

Keywords International environmental agreements · Breakthrough technology

JEL Classification Q28 $\cdot$ C72 $\cdot$ F42

\section{Introduction}

In a recent paper, Barrett (2006) investigates whether an international environmental agreement, such as a climate treaty, can perform better if it relies directly on targeted R\&D and the adoption of breakthrough technologies. This is important because the literature shows that treaties that just rely on abatement, such as the Kyoto Protocol, cannot be expected to

\footnotetext{
This article was initiated during our participation in the 'Environmental economics: policy instruments, technology development and international cooperation' project at the Centre for Advanced Study (CAS) at the Norwegian Academy of Science and Letters in Oslo in 2005-2006. We are grateful for many useful comments and suggestions from the participants. We are also grateful for comments on earlier versions of this paper given by Geir Asheim, Scott Barrett, Bard Harstad, Chuck Mason, participants of the NBER Summer Institute, and two anonymous referees.
}

M. Hoel $(\varangle)$

Department of Economics, University of Oslo, P.O. Box 1095, Blindern, 0317 Oslo, Norway

e-mail: m.o.hoel@econ.uio.no

A. de Zeeuw

Department of Economics and TSC, Tilburg University, P.O. Box 90153, 5000 LE Tilburg,

The Netherlands

e-mail: a.j.dezeeuw @uvt.nl 
achieve much (Barrett 2003; Finus 2003). Furthermore, the international debate circles to some extent around the question whether international treaties should focus on technology development rather than on emission reduction. Barrett (2006) reaches the conclusion that in general the answer to the question whether such a treaty can perform better is no, with the exception of breakthrough technologies that exhibit increasing returns to scale. In this paper we will show that the picture is not necessarily so grim. By introducing the option that the adoption costs of a breakthough technology vary with the level of R\&D, we will show that a large stable coalition can result that leads to a substantial improvement in average welfare. The basic idea is that a coalition can improve on a non-cooperative equilibrium in which the countries invest sufficiently much in the public good R\&D to trigger a breakthrough technology. Either a coalition forms that invests even more in $R \& D$ (accepting that the other countries free-ride on this investment) because it pays to further lower the costs of adoption. Or a coalition forms that invests less in $R \& D$ but is large enough to realize a substantial improvement in average welfare by itself. In the last case the non-cooperative equilibrium would lead to an overinvestment in R\&D.

The literature on international environmental agreements has investigated various approaches for enlarging the size of the stable coalition. If coalition members can secure extra positive externalities between them, for example by linking the agreement to an $R \& D$ agreement with larger spillovers between coalition members, the size of the stable coalition will grow (Carraro and Siniscalco 1997). In general, if the issue of negotiation can be linked to a second issue, the bargaining space may get larger with more opportunities to reach an agreement (Cesar and de Zeeuw 1997). Another approach is extending the stability concept. For example, the concept of farsightedness takes into account that countries consider further defections as a consequence of their own defection and this stabilizes larger coalitions (Diamantoudi and Sartzetakis 2002). A similar effect is achieved by adding a first stage to the game in which countries decide on a minimum participation constraint, because this also prevents one-by-one defections down to the standard small-size stable coalition (Carraro et al. 2009). Finally, learning in case of uncertainty can enlarge the size of the stable coalition as well (Kolstad 2007). This paper takes another angle by focusing on R\&D and the adoption of breakthrough technologies. Cooperation may not be needed to achieve sufficient R\&D and a switch to the new technology but cooperation may be needed to prevent under- or overinvestment in R\&D.

\section{Adoption Costs, R\&D and the Social Optimum}

Barrett (2006) assumes a fixed cost of adoption, $c$, which requires a certain level of R\&D, $M$. In the R\&D phase countries decide how much they want to contribute to this public good. In his approach the R\&D phase precedes coalition formation and the adoption of new technology. If the expected average net benefits are sufficiently high, in the R\&D phase an equilibrium exists that yields the required $\mathrm{R} \& \mathrm{D}$ level $M$. The reason is simply that each country does not want to contribute less, because then the benefits are not realized, but does not want to contribute more either, because then the costs become higher without higher expected benefits. This is an example of voluntary provision of a public good (see e.g. Bagnoli and Lipman 1989).

We change the set-up somewhat and link the cost of adoption $c$ to the level of R\&D $M$ by a function $c=c(M)$ with $c^{\prime}<0, c^{\prime \prime}>0$. In this way a trade-off is introduced between $\mathrm{R} \& \mathrm{D}$ costs and costs of adoption. The idea is that the more research is done the lower are the expected future costs of using this technology. Examples of the type of technologies we 
are thinking of are Carbon Capture and Storage (CCS), capturing $\mathrm{CO}_{2}$ from air (see e.g. Lackner et al. 2001, and Pielke 2009), and developing small batteries for electrical cars. In case of cooperation, groups of countries may set up research joint ventures with the purpose to develop these technologies, and the more they invest the lower the costs will be of implementing these technologies. For instance, smaller batteries require more R\&D but reduce the costs of implementing electrical cars, and increased energy efficiency of CCS or air capture requires more $\mathrm{R} \& \mathrm{D}$ but reduces the operating costs of these technologies. We assume that for values of $M$ giving $c(M) \leq 1, c(M)+M$ is increasing in $M$, i.e. $-c^{\prime}<1$. This last assumption is reasonable: If the opposite were true, one dollar of investment in this type of R\&D would reduce the adoption costs for this technology by more than one dollar for all countries.

In our setting it makes sense to position coalition formation before the choice of $R \& D$, so that decisions will balance $R \& D$ costs and adoption costs. This implies that we change the order of decisions as compared to Barrett (2006): countries first decide whether they want to join the coalition or not before they decide on investment in R\&D and adoption. We will show below that the change in the order of decisions does not affect the qualitative results. One of the cases below is not much different from Barrett's model and also leads to a stable coalition that does not achieve much. The only difference in that case is that in our setting $R \& D$ costs are borne by the coalition, whereas Barrett (2006) assumes that R\&D is financed by all countries not knowing whether they will be in or out of the coalition. Qualitative differences occur in the other cases and are only driven by the assumption that adoption costs depend on $R \& D$ expenditures.

Barrett (2006) combines the option of adopting a breakthrough technology with a standard international abatement game which can be seen as a choice between a new clean technology and abatement options within the context of the old technology. The last situation is in fact a default option and we abstract from that by focusing only on the choice of adopting the new technology or not. It is easy to show that by doing this the qualitative results remain the same, but in this way the analysis becomes more transparent. As in Barrett (2006) we assume that R\&D is a public good, but the same results can be derived in a setting with imperfect R\&D spillovers. The countries are assumed identical, but it would of course be interesting to extend the analysis to a heterogeneous set of countries; this is left for further research. Finally, our model is deterministic, but it would of course also be interesting to extend the analysis to uncertainty and learning by doing.

We normalise the benefits for a single country of adopting the new technology to 1 . This is equal to the damage (for the single country) caused by the pollution that will be generated continuing with the old technology (for example the damage caused by further emitting large amounts of $\mathrm{CO}_{2}$ ). These benefits can be realized by the country itself but can also be freerider benefits from adoption of the new technology in another country. Once the technology is developed, it is individually rational for each country to adopt if and only if $c \leq 1$. The problem is that usually we have that $c(M)+M>1$ for all $M$, so that it is not optimal for an individual country to develop and adopt the new technology just by itself. In the setting with a constant $c$ that Barrett (2006) uses, $c>1$ simply means that without coalition formation the new technology will not be adopted: a group of countries is needed to generate sufficient benefits to cover the costs of adoption. Note that this is similar to the standard linear international abatement game (e.g. Kolstad 2007). In our setting, however, it is in principle possible that the countries together invest so much in R\&D that it becomes individually rational to adopt the new technology, because $c$ depends on $M$. We denote that level by $\bar{M}$, which means that $c(\bar{M})=1$.

If the countries operate together, they minimize $N c(M)+M$, and if the resulting costs are sufficiently low to make it collectively rational to adopt the new technology, each country will have a net benefit equal to 


$$
V(N)=N-\min _{M}\left[c(M)+\frac{M}{N}\right]
$$

We assume that $V(N)$ is positive so that the social optimum leads to full adoption.

\section{Non-Cooperative Equilibria}

Without any cooperation, the outcome is given as a subgame perfect equilibrium of a two-stage game. In the first stage each country chooses its level of R\&D investments. The properties (i.e. the costs) of the new technology are determined by the sum of R\&D investments as explained in Sect. 2. In the second stage each country chooses whether to adopt the new technology. It is clear from our assumptions that one subgame perfect equilibrium of this game is for all countries to have no R\&D investment, and thus no adoption of any new technology. However, there may exist a second subgame perfect symmetric equilibrium. This will be the case if $\bar{M}$ exists and $\bar{M} \leq N(N-1)$. In this case an equilibrium exists in which each of the $N$ countries invests $\bar{M} / \bar{N}$ in R\&D, and all countries adopt the new technology. Each country will in this case have a net benefit equal to

$$
\tilde{V}=N-1-\frac{\bar{M}}{N}
$$

which is non-negative for $\bar{M} \leq N(N-1)$. It will not be beneficial for a country to deviate unilaterally since the total level of R\&D will end up below $\bar{M}$, so that none of the countries will adopt the new technology anymore. Moreover, since we have assumed that $c(M)+M$ is increasing in $M$ it does not pay for an individual country to increase investments above $\bar{M}$. We can thus conclude that when $\bar{M} \leq N(N-1)$, a symmetric Nash equilibrium in R\&D investments with full adoption exists. Notice that this game also has asymmetric equilibria, but we do not analyze these. Furthermore, notice that even though we have full adoption of the new technology in this equilibrium, the outcome differs from the first-best social optimum. In the latter, the choice of $R \& D$ was determined so that the sum of investment and adoption costs for each country was minimized. In the non-cooperative outcome, the level of R\&D is exactly as large as it must be for all countries to want to adopt the new technology. This level will generally differ from the first-best R\&D level, and generally we do not know whether it is lower or higher than the first-best level.

Since even this second non-cooperative equilibrium (if it exists) is inferior to the social optimum, we want to investigate if a stable coalition can improve upon the outcome compared to the non-cooperative equilibria. We therefore now turn to coalition formation and show that substantial improvement is possible in case the non-cooperative equilibrium with full adoption exists.

\section{Coalition Formation}

We use the model for coalition formation that was introduced into the literature on international environmental agreements by Hoel (1992), Carraro and Siniscalco (1993), and Barrett (1994), and which is based on a model for cartel stability developed by d'Aspremont et al. (1983). The idea is that countries first decide whether they want to join the coalition or not before they take action. In the equilibrium of this two-stage game a member of the coalition 
does not have an incentive to quit (internal stability) and an outsider does not have an incentive to join (external stability).

This literature usually provides a rather grim picture: Stable coalitions are large if there is not much to gain from cooperation and are otherwise typically very small (Barrett 1994). This result was challenged from different angles. Chander and Tulkens (1995), for example, use the gamma-core concept from cooperative game theory to show that the grand coalition is stable in the sense of being an element of the core. The basic idea is very similar to the idea of trigger strategies in repeated games, where the threat of loosing cooperative benefits prevents countries to cheat. The essential difference with the standard literature on international environmental agreements is the assumption on the behaviour of the other coalition members in case a country defects. In the stability concept above, the remaining coalition stays intact, whereas in the gamma-core concept the original coalition falls apart in case a country (or a group of countries) defects. Diamantoudi and Sartzetakis (2002) apply the idea of farsightedness to show that a set of stable coalitions exists, among which large ones. The basic idea is that if a country defects, other coalition members defect as well, but only up to the point where the remaining coalition is stable again. This threat proves to be sufficient to sustain large coalitions, although in a dynamic context, where detection of deviations takes time, the idea may not work (de Zeeuw 2008).

In this paper we base ourselves on the concept of internal and external stability, because the underlying assumption seems reasonable for the current practice of international environmental agreements. We will show, however, that if we replace the second stage of this game by the two-stage game of investment and adoption, decribed in the previous section, a large stable coalition may arise. We distinguish two cases.

Case 1: $\bar{M}>N(N-1)$

It is immediately clear that if the non-cooperative equilibrium with full adoption $(M=\bar{M})$ does not exist, outsiders will not invest in R\&D. In that case $\tilde{V}$ is negative, so that outsiders do not have an incentive to invest in R\&D if the coalition does not invest more than $k \bar{M} / N$ in total. The coalition could consider to invest more in R\&D in order to induce the outsiders to invest as well, but then the coalition's net benefit becomes negative. This implies that the coalition is on its own, and adopts the new technology if and only if $c(M) \leq k$. Individual outsiders will not adopt, since total R\&D expenditures $M$ will be smaller than $\bar{M}$. Formally, we have the following three-stage game:

Stage 1: each country chooses whether or not to join the coalition

Stage 2: the coalition chooses R\&D expenditures

Stage 3: the coalition chooses whether or not to adopt the new technology.

Note that there is no new stage of coalition formation between stages 2 and 3: Once a coalition is formed in stage 1 , it remains a coalition after the investment decision has been made in stage 2 .

Suppose that a coalition of size $k$ forms, $0<k<N$. Similar to the full-cooperative case in the previous section, the coalition minimizes $k c(M)+M$, and if the resulting costs are sufficiently low to make it collectively rational to adopt the new technology, each member of the coalition will have a net benefit equal to

$$
\phi(k)=k-\min _{M}\left[c(M)+\frac{M}{k}\right]
$$


The solution to the minimization in (3) is denoted by $M^{*}(k)$, and $c^{\prime \prime}>0$ implies that $M^{*}$ is increasing in $k$. Note that the decision to join the coalition or not must indeed be binding after the second stage. Otherwise, if countries could choose again after the R\&D investments are made, an individual country would prefer to be outside the coalition of size $k-1$, because the adoption costs are larger than 1 in the case we are now considering.

Since $\phi(k)$ is increasing in $k$ (using the envelope theorem) and since we have assumed that $\phi(1)$ is negative and $\phi(N)=V(N)$ is positive, $\phi(k)$ must be 0 for some $k^{0}$ between 1 and $N$. Consider the coalition of size $K^{0}$, defined as the smallest integer at least as high as $k^{0}$. The net benefit to each member of this coalition is $\phi\left(K^{0}\right)$, which is non-negative but small. This coalition is internally stable: if one country instead of joining the coalition chooses to be an outsider, the coalition of size $K^{0}-1$ will not develop the new technology, so that it is better to stay in the coalition. Consider now the coalition of size $K$ satisfying $k^{0}+1 \leq K \leq N$. The net benefit to each member of such a coalition is $\phi(K)$, which is smaller than $K-1$ since $c\left(M^{*}(K)\right)>1$. Therefore this coalition is not internally stable: if one country instead of joining the coalition chooses to be an outsider, the coalition of size $K-1$ will develop and adopt the new technology, so that the country will get a benefit of $K-1$ without costs. Consequently, the coalition of size $K^{0}$ is also externally stable and thus the only stable coalition.

Note that the stable coalition achieves very little. This is in line with the pessimistic result that Barrett (2006) derives. In this case our model is only slightly different. We allocate total $R \& D$ costs to the coalition, whereas Barrett (2006) assumes that $R \& D$ is financed by all the countries not knowing whether they will be in or out of the coalition. Otherwise, the models are essentially the same in this case. However, in our setting a non-cooperative equilibrium with full adoption $(M=\bar{M})$ may exist and then the picture changes.

Case 2: $\bar{M} \leq N(N-1)$

If the non-cooperative equilibrium with full adoption $(M=\bar{M})$ exists, the coalition has a choice to dissolve again and rely on this equilibrium, with a non-negative net benefit $\tilde{V}$ for its members, or to act as a coalition. If the coalition acts as a coalition, then either the coalition plays the investment game in the R\&D phase with the outsiders or the coalition is on its own in that phase. Although this investment game has equilibria in which a coalition member invests less than or more than an outsider, we disregard these equilibria, just as we did not consider asymmetric non-cooperative equilibria in the previous section. This implies that the coalition either relies on the non-cooperative equilibrium with full adoption $(M=\bar{M})$, or improves on that by acting as a coalition and doing all the investment on its own. Formally, we have the following four-stage game now:

Stage 1: each country chooses whether or not to join the potential coalition

Stage 2: the potential coalition decides whether or not to act as a coalition

Stage 3: either each individual country or the coalition chooses R\&D expenditures

Stage 4: either individual countries or coalition and outsiders choose whether or not to adopt the new technology.

Compared with the case studied previously (with $\bar{M}>N(N-1)$ ), stage 2 is new. However, stage 2 was not relevant in the previous case, since in that case there could be no disadvantages from acting as a coalition.

We first focus on stages 3 and 4 of the game in case the coalition stays intact and chooses R\&D expenditures. The situation is more complicated than above because the coalition of size $k$ may choose $M$ to be equal to or larger than $\bar{M}$. Three possibilities occur. The first 
Fig. 1 Value function of a coalition member as a function of the coalition size

Fig. 2 Investments in $\mathrm{R} \& \mathrm{D}$ as a function of the coalition size
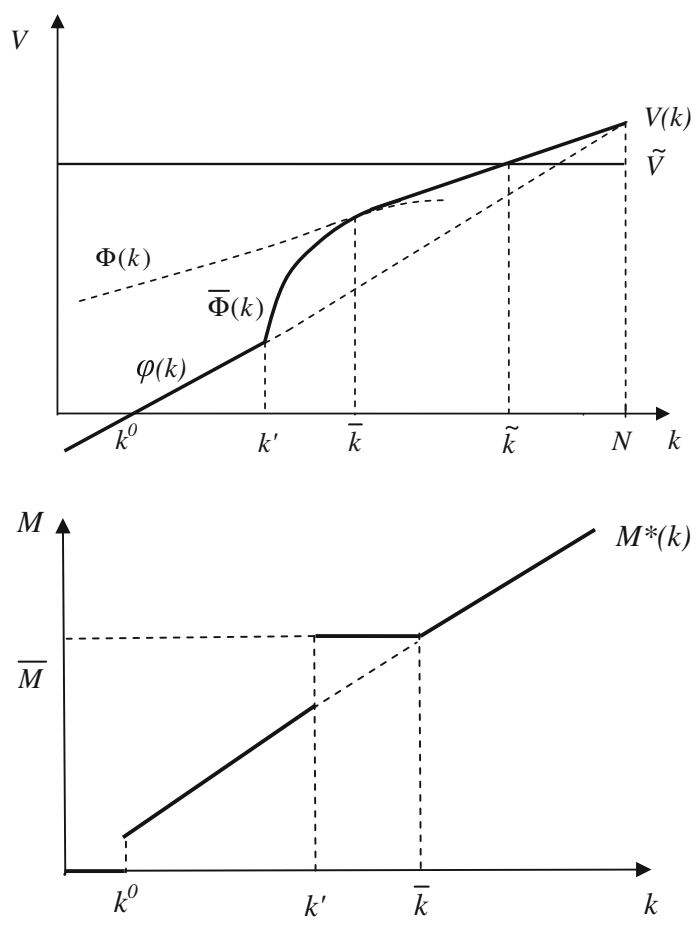

possibility is that the optimal investment in R\&D is sufficiently high so that all countries adopt the new technology, which means that each member of the coalition will have a net benefit equal to

$$
\Phi(k)=N-\min _{M}\left[c(M)+\frac{M}{k}\right]
$$

The second possibility is that the coalition invests $\bar{M}$ at its own cost in order to induce the outsiders to adopt the new technology as well, which means that each member of the coalition will have a net benefit equal to

$$
\bar{\Phi}(k)=N-\left[1+\frac{\bar{M}}{k}\right]
$$

The final possibility is that it does not pay for the coalition to invest so much, which puts us back in the situation above with $\phi(k)$, given by (3), as the net benefit. The value function of a member of the coalition of size $k$ in this case is simply the maximum of the three possibilities $\Phi(k), \bar{\Phi}(k)$ and $\phi(k)$ and is denoted by $V(k)$. Two typical outcomes are depicted in Figs. 1 and 3 with the corresponding levels of R\&D in Figs. 2 and 4, respectively. In Figs. 1 and 2 $M^{*}(N)$ is larger than or equal to $\bar{M}$, so that all three possibilities can occur, and in Figs. 3 and $4 M^{*}(N)$ is smaller that $\bar{M}$, so that only the last two possibilities can occur.

It is easy to verify that $\tilde{V}>\bar{\Phi}(k)$ for all $k<N$, with $\tilde{V}$ given by (2). In other words, the net benefit of a country in the non-cooperative equilibrium with $M=\bar{M}$ exceeds the net benefit of a member of a coalition investing $\bar{M}$ in R\&D. This is illustrated in Figs. 1 and 3. The intersection point between $V(k)$ and $\tilde{V}$ is denoted by $\tilde{k}$. It follows that for $k<\tilde{k}$ the coalition 
Fig. 3 Value function of a coalition member as a function of the coalition size

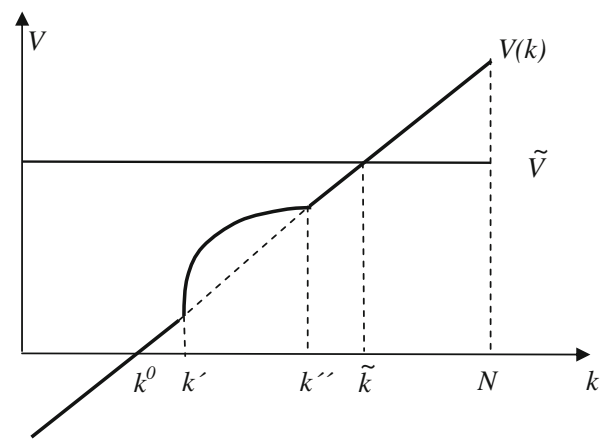

Fig. 4 Investments in R \& D as a function of the coalition size

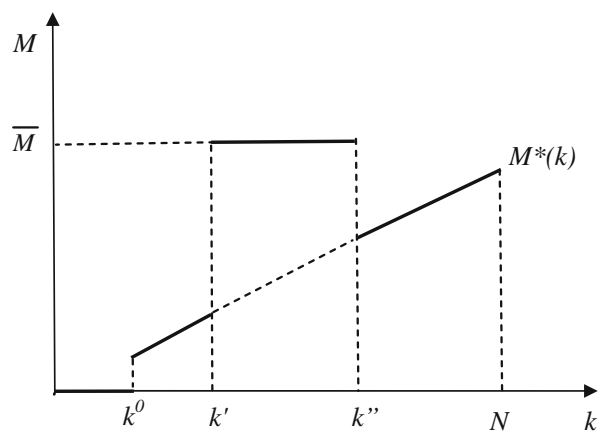

cannot do better on its own, compared to the non-cooperative equilibrium. Hence, a coalition of this size decides in stage 2 of the game not to act as a coalition, so that the symmetric non-cooperative equilibrium with $M=\bar{M}$ and with net benefits equal to $\tilde{V}$ results. For $k>\tilde{k}$, however, the coalition will invest $M^{*}(k)$ in R\&D on its own and adopt the new technology, giving its members a net benefit $V(k)>\tilde{V}$. If $M^{*}(k)>\bar{M}$, as in Fig. 1, outsiders will adopt the new technology as well, and each of them achieves a net benefit equal to $N-c\left(M^{*}(k)\right)$. If $M^{*}(k)<\bar{M}$, as in Fig. 3, outsiders will not adopt the new technology, and each of them receives a benefit equal to $k$. Outsiders are always better off than coalition members, so that both coalition members and outsiders are better off than in the non-cooperative equilibrium with full adoption.

Define $\tilde{K}$ as the lowest integer satisfying $\tilde{K} \geq \tilde{k}$. A coalition of size $\tilde{K}-1$ will not form in stage 2 of the game. It is in the interest of those countries to stay apart and to end up in the non-cooperative equilibrium because $\tilde{V}>V(\tilde{K}-1)$. This implies that a coalition of size $\tilde{K}$ is internally stable, because leaving this coalition will give a net benefit $\tilde{V}$ which is smaller than $V(\tilde{K})$.

Under reasonable conditions, a coalition of size $K \geq \tilde{k}+1$ is not internally stable. For the situation of Figs. 3 and 4, it is easy to see that a coalition of size $K \geq \tilde{k}+1$ is never internally stable: An outsider to the coalition of size $K-1$ is better off than a member of the coalition of size $K$, since the costs of adoption are higher than 1 (which is the benefit of one more country adopting the technology), and the outsider is not investing in $\mathrm{R} \& \mathrm{D}$. It follows that in this situation the coalition of size $\tilde{K}$ is also externally stable, and thus the largest stable coalition.

In the situation of Figs. 1 and 2, it is not quite so obvious that an outsider to the coalition of size $K-1$ is better off than a member of the coalition of size $K$, provided $K \geq \tilde{k}+1$. 
In this case full adoption occurs both for the coalition of size $K-1$ and $K$, and the difference in payoffs is thus only in costs. The cost of an outsider to the coalition of size $K-1$ is simply $c\left(M^{*}(K-1)\right)$, while the cost of a member of the coalition of size $K$ is $c\left(M^{*}(K)\right)+\frac{M^{*}(K)}{K}$. Consequently, a coalition of size $K \geq \tilde{k}+1$ is internally stable if and only if

$$
c\left(M^{*}(K-1)\right) \geq c\left(M^{*}(K)\right)+\frac{M^{*}(K)}{K}
$$

or

$$
c\left(M^{*}(K-1)\right)-c\left(M^{*}(K)\right) \geq \frac{M^{*}(K)}{K}
$$

For this inequality to hold, adoption costs must decline quite significantly if the coalition is enlarged by one member. From the definition of $M^{*}$, given by (3), it is clear that

$$
c\left(M^{*}(K-1)\right)+\frac{M^{*}(K-1)}{K-1} \leq c\left(M^{*}(K)\right)+\frac{M^{*}(K)}{K-1}
$$

implying that the 1.h.s. of (6) cannot exceed $\frac{1}{K-1}\left[M^{*}(K)-M^{*}(K-1)\right]$. A necessary condition for (6) to hold is therefore that

$$
\frac{M^{*}(K)-M^{*}(K-1)}{M^{*}(K)} \geq \frac{K-1}{K}
$$

If for instance $K=10$, the decline in R\&D investment when the coalition size drops from 10 to 9 must be at least $90 \%$. Although such cases cannot theoretically be ruled out, we find them quite implausible. Under reasonable conditions, a coalition of size $K \geq \tilde{k}+1$ is therefore not internally stable; the coalition of size $\tilde{K}$ is thus the largest stable coalition.

The coalition $\tilde{K}$ gives its members only a slightly higher net benefit than they would have achieved in the non-cooperative equilibrium with $M=\bar{M}$. In contrast, the outsiders get a much higher net benefit in this case; the average net benefit may therefore be substantially higher than in that non-cooperative equilibrium. The uneven distribution of benefits is typical for these models and requires further attention (just as the uneven distribution in the theoretical equilibrium for the Ultimatum game). In this paper, however, we focus on what coalition formation can achieve in terms of average welfare. Before discussing the results, we first give a numerical illustration.

\section{A Numerical Illustration}

Suppose that the function for adoption costs is given by

$$
c(M)=\frac{\gamma}{M}
$$

This implies that

$$
M^{*}(k)=\sqrt{\gamma k}
$$

and

$$
\phi(k)=k-2 \sqrt{\frac{\gamma}{k}}
$$


Fig. 5 Value function of a coalition member as a function of the coalition size
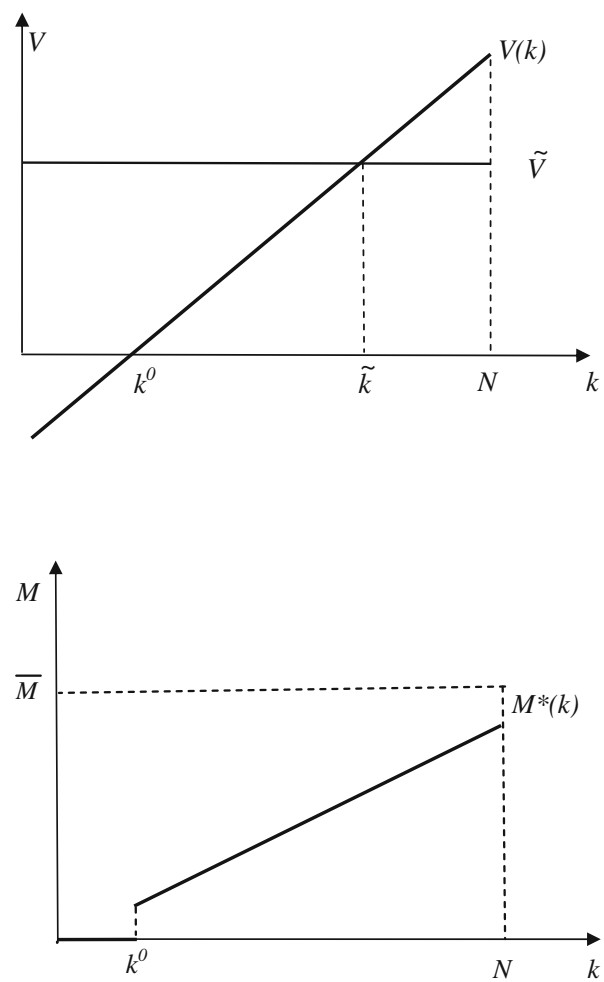

Fig. 6 Investments in R \& D as a function of the coalition size

Suppose that the number of countries $N=20^{1}$ and that $\gamma=225$. It follows that the first-best R\&D investment level is 67 , giving each country a net benefit equal to 13.29 . Furthermore, $\bar{M}=\gamma=225$, so that a non-cooperative equilibrium with positive R\&D exists, giving each country a net benefit equal to 7.75 . In this case the non-cooperative equilibrium with $M=\bar{M}$ leads to an overinvestment in $\mathrm{R} \& \mathrm{D}$, implying that this net benefit is considerably lower than the one in the social optimum.

Since $M^{*}(N)<\bar{M}$, Figs. 3 and 4 apply. It is easily verified that in this example the value function $V(k)$ does not have the kinks depicted in Fig. 3, but yields the simpler Figs. 5 and 6. The smallest coalition giving its members a non-negative net benefit is $K^{0}=10$. For this coalition size, coalition members get a net benefit equal to 0.51 , while outsiders get 10. The average net benefit is 5.26 , which is considerably lower than the net benefit in the social optimum and also lower than the net benefit in the non-cooperative equilibrium with $M=\bar{M}$. The largest stable coalition size in our setting, however, is $\tilde{K}=16$, giving coalition members a net benefit equal to 8.5 . The 4 outsiders get a net benefit equal to 16 , so that the average net benefit is 10 . Although this is smaller than the average net benefit in the social optimum, it is considerably larger than for the coalition of size $K^{0}$, and also larger than the average net benefit in the non-cooperative equilibrium with $M=\bar{M}$.

1 In 2005 the 20 countries with the largest carbon emissions from energy use stood for $88 \%$ of total emissions (counting the EU as one country). 


\section{Conclusion}

International environmental agreements are usually viewed upon as institutions with the purpose to overcome negative transboundary externalities. Due to free-rider incentives, however, treaties with a large number of signatories cannot be expected to be stable, so that a large part of the benefits of cooperation cannot be achieved. Barrett (2006) extended this framework to collective financing of R\&D and adoption of breakthrough technologies, but effectively reaches the same conclusion, unless these technologies exhibit increasing returns to scale. In this paper we connect the level of R\&D to the cost of adoption, and argue first that non-cooperative behaviour may lead to full adoption with a sufficiently high level of the public good R\&D. This does not mean, however, that international treaties are not needed anymore. A treaty can do better, as it can either invest more in R\&D in order to reduce the cost of adoption, or it can prevent overinvestment in R\&D. We reach the important conclusion that in this setting the stability properties of international environmental agreements are much better than in the setting where treaties only focus on emission reduction.

Our result implies that it can indeed be beneficial for international environmental agreements to consider breakthrough technologies and R\&D whenever this is appropriate, not only for the reason Barrett (2006) puts forward, but also for reasons put forward in this paper. For example, the Kyoto Protocol could consider setting up an R\&D joint venture in order to develop carbon-free energy technologies instead of leaving this to the individual countries. Either a cheaper technology, adopted by all countries, will be developed, or a more expensive one, adopted by the signatories only. In both cases global welfare will increase. The first best will not be achieved, but the size of the coalition will be large and substantial benefits of cooperation will be realized.

Open Access This article is distributed under the terms of the Creative Commons Attribution Noncommercial License which permits any noncommercial use, distribution, and reproduction in any medium, provided the original author(s) and source are credited.

\section{References}

Bagnoli M, Lipman B (1989) Provision of public goods: fully implementing the core through private contributions. Rev Econ Stud 56:583-601

Barrett S (1994) Self-enforcing international environmental agreements. Oxf Econ Pap 46:878-894

Barrett S (2003) Environment and statecraft: the stategy of environmental treaty-making. Oxford University Press, Oxford

Barrett S (2006) Climate treaties and "breakthrough" technologies. Am Econ Rev 96(2):22-25

Carraro C, Siniscalco D (1993) Strategies for the international protection of the environment. J Public Econ 52(3):309-328

Carraro C, Siniscalco D (1997) R\&D cooperation and the stability of international environmental agreements. In: Carraro C (ed) International environmental negotiations: strategic policy issues. Edward Elgar, Cheltenham pp 71-96

Carraro C, Marchiori C, Oreffice S (2009) Endogenous minimum participation in international environmental treaties. Environ Resour Econ 42(3):411-425

Cesar H, de Zeeuw A (1997) Issue linkage in global environmental problems. In: Xepapadeas A (ed) Economic policy for the environment and natural resources. Edward Elgar, Cheltenham, pp 158-173

Chander P, Tulkens H (1995) A core-theoretic solution for the design of cooperative agreements on transfrontier pollution. Int Tax Public Finance 2:279-293

d'Aspremont C, Jacquemin A, Gabszewicz J, Weymark J (1983) On the stability of collusive price leadership. Can J Econ 16(1):17-25

de Zeeuw A (2008) Dynamic effects on the stability of international environmental agreements. J Environ Econ Manag 55(2):163-174 
Diamantoudi E, Sartzetakis E (2002) International environmental agreements - the role of foresight. mimeo

Finus M (2003) Stability and design of international environmental agreements: the case of transboundary pollution. In: Folmer H, Tietenberg $\mathrm{T}$ (eds) The international yearbook of environmental and resource economics 2003/2004. Edward Elgar, Cheltenham, pp 82-158

Hoel M (1992) International environmental conventions: the case of uniform reductions of emissions. Environ Resour Econ 2(2):141-159

Kolstad C (2007) Systematic uncertainty in self-enforcing international environmental agreements. J Environ Econ Manag 53(1):68-79

Lackner K, Grimes P, Ziock H-J (2001) Capturing carbon dioxide from air. In: Proceedings of the first national conference on carbon sequestration, Washington, DC, USA

Pielke RA Jr (2009) An idealized assessment of the economics of air capture of carbon dioxide in mitigation policy. Environ Sci Policy 12:216-225 